\title{
Design e Cultural driven innovation
}

\section{Sommario:}

Il saggio si propone di inquadrare il concetto di Cultural Driven Innovation nel discorso su design e patrimonio culturale contemporaneo, inteso come l'heritage continuum attivabile dal design tramite le tecnologie digitali e mobile. In questo approccio il Cultural Heritage non è considerato solo leva per uno sviluppo più sostenibile ma vera e propria fonte di innovazione, grazie al suo ri-uso creativo non soltanto in nuovi prodotti culturali fruibili da utenti finali, ma in strategie e strumenti, abilitati dal design, di co-creazione di valore per tutta la filiera.

Parole chiave: Cultural Driven Innovation, Heritage continuum, Phygital, Attivazione del patrimonio, Ri-uso del patrimonio

\section{Design and Cultural driven innovation}

\section{Abstract}

The essay aims to frame the concept of Cultural Driven Innovation in the discourse on design and contemporary cultural heritage, understood as the heritage continuum that can be activated by design through digital and mobile technologies. In this approach, Cultural Heritage is not only considered a lever for a more sustainable development but a real source of innovation, thanks to its creative re-use not only in new cultural products usable by end users, but in strategies and tools, enabled by design, of co-creation of value for the whole $\mathrm{CH}$ chain.

Keywords: Cultural Driven Innovation, Heritage continuum, Phygital, Attivazione del patrimonio, Ri-uso del patrimonio 


\section{Design e cultura ai tempi dell'heritage continuum}

Il design ha da sempre a che fare con l'intangibile, dovendosi ad esempio confrontare con la progettazione di processi, funzioni, significati legati agli oggetti con cui quotidianamente interagiamo ed oggi la componente immateriale del progetto diventa sempre più rilevante. Non solo perché le tendenze relative alla trasformazione digitale hanno modificato il concetto stesso di cultura materiale: l'utilizzo delle tecnologie ed in particolare il concetto di phygital ('interazione tra il mondo fisico e quello digitale) stratificano una geografia diffusa di azioni e processi di materializzazione, che rendono possibile un'esperienza profonda, densa e intensa di prodotti, estesa nel tempo e nello spazio, per cui si può parlare di materialità aumentata o di meta-materialità. Ma soprattutto perché, all'interno di confini e ambiti di ricerca e intervento sempre più sfumati, tali trasformazioni continuano costantemente ad ampliare gli ambiti di applicazione del design, dalla tradizionale produzione di beni materiali, e dalla ormai consolidata progettazione di strategie, comunicazione, processi, servizi ed esperienze, alla concezione di policies, identità, rappresentazioni e conversazioni. La cultura del design contemporaneo sta sempre più permeando, nell'ambito della produzione culturale e scientifica, sia gli ampi scenari politici e socio-economici che le estetiche e gli immaginari del quotidiano, con le sue molteplici prassi. In questo quadro, le implicazioni di tipo culturale, sociale, politico ed economico del design, sono strettamente collegate alla capacità di generare visioni e approcci in cui la tecnologia incorpora la cultura e la cultura incorpora la tecnologia, evitando un punto di vista meramente tecno-centrico e promuovendo piuttosto un nuovo umanesimo socio-tecnico, percepibile e materializzabile in diverse scale e luoghi (geografico, produttivo...).

L'ambito specifico del design per la valorizzazione del cultural heritage è un caso emblematico e paradigmatico di questa necessaria evoluzione disciplinare, avendo come oggetto di progetto delle entità che sostanziandosi in evidenze materiali date (dall'opera d'arte, al monumento, al bene demo-antropologico, al bene archivisticolibrario, alle collezioni e ai musei, etc.) sono "progettabili" nella loro dimensione immateriale di valore da riconoscere, salvaguardare, comunicare, fruire. Progettare in ambito culturale oggi richiede inoltre una nuova consapevolezza anch'essa legata alla rivoluzione digitale: l'heritage grazie all'uso delle tecnologie si connota sempre più come un continuum inscindibile tra materiale e immateriale, accessibile e fruibile in maniera diffusa, personalizzabile e condivisa, attraverso una ecologia di contenuti che collegano le collezioni di musei, istituzioni culturali, archivi, repository on line e territori in uno spazio fluido e stratificato tra fisico e virtuale.

In questo continuum rientrano, accanto alle forme autorevoli e riconosciute istituzionalmente di Cultural Heritage, anche nuove espressioni come memorie, storie e oggetti, pratiche estetiche, produzione culturale della società contemporanea che possono essere definite forme di Contemporary Heritage (Battesti, 2012) e che pur nella loro ancora problematica legittimazione istituzionale, avendo una notevole attualità e presenza nella vita delle persone, possono diventare una delle chiavi di accesso per riannodare i legami spesso allentati tra heritage ed esperienza quotidiana, rendendo più comprensibile e praticabile anche il patrimonio più autorevole.

È proprio questo contesto dell'heritage continuum (Ozdil, 2014; Lupo, Vitale, 2017) a rappresentare la sfida contemporanea del design per il patrimonio culturale, in quanto richiede di essere approcciato con una rinnovata visione che integra cultura e tecnologie, capace di legare le eredità culturali con le dinamiche di sviluppo in modo realmente innovativo e culturalmente e socialmente sostenibile. 


\section{Attivare il cultural heritage tramite il design come leva per lo sviluppo.}

Il design per la valorizzazione del cultural heritage è territorio fertile di esperienze progettuali che lavorano su dimensioni intangibili e utilizzano un approccio in cui tecnologia e cultura sono fortemente integrate.

Ambiti quali beni culturali autorevoli sono stati oggetto di ricerca e pratica progettuale da parte della comunità accademica italiana del design (ed in particolare quella milanese) sin dall'inizio degli anni 2000 con una serie di pubblicazioni seminali (Seassaro, 2003; Celaschi,Trocchianesi, 2004) e una ricerca fondativa quale d.Cult. Il design per la valorizzazione dei beni culturali (Maffei, Parente, Villari, 2006) poi confluita in successive pubblicazioni (Parente, Lupo 2009): in queste esperienze emerge il forte approccio culturale con cui anche la componente tecnica e tecnologica sono orientate per lavorare su dimensioni immateriali quali la messa in valore del patrimonio attraverso modelli relazionali e territoriali, la sua fruizione, la partecipazione e l'empowerment delle comunità.

Anche a livello internazionale, la con la conferenza Cumulus 2005 di Lisbona dal titolo Pride and pre-design. The cultural heritage and the science of design si tessono analoghi importanti legami tra il patrimonio culturale e la cultura progettuale (Corte Real et al., 2005): il design consente una continua rigenerazione del patrimonio (Vasconcellos 2005; Chih-Yuan, 2005) salvaguardando le diversità locali attraverso l'acquisizione, rappresentazione e appropriazione della conoscenza tacita dei luoghi e delle produzioni tipiche (Wood, 2005; Pombo, 2005; Cabrera-Viancha, 2005) facendo dialogare tradizione e memoria con l'identità contemporanea (Perrone, Buchler, 2005).

Parallelamente il network di ricerca Nodem (https://nodem.org/), con le sue conferenze internazionali, ha focalizzato l'attenzione sul tema delle tecnologie per il patrimonio culturale, che hanno lo scopo di migliorare l'esperienza dei fruitori e il loro coinvolgimento o aumentare l'interazione sociale e le capacità di interpretazione del patrimonio. In questo filone, una serie di sperimentazioni e studi, a partire da musei narrativi (Studio Azzurro, 2011) e spazi performativi (Dernie, 2006), applicano le potenzialità delle tecnologie digitali per musei e patrimonio culturale (Allen, Lupo 2012) per offrire esperienze di tipo inclusivo, dialogico e partecipativo di co-creazione del patrimonio (Affleck, Kvan, 2008; Kuo Wai Tchen, Ševcenko 2011; Salgado, Marttila, 2013; Simon, 2010; Giaccardi 2012; Smith and Iversen, 2014).

Da tutte queste esperienze è evidente che la maturità raggiunta dall'ambito progettuale dei beni culturali è principalmente espressa dall'arricchire il tradizionale concetto di valorizzazione con modelli capaci di "attivare", grazie al design, il cultural heritage come leva per lo sviluppo e produrre quindi uno sviluppo cultural driven, in linea con le indicazioni dell'OCSE sul cultural oriented development (Greffe, 2005). Sostanzialmente si tratta di affiancare al progettare per il patrimonio culturale, il progettare con il patrimonio culturale: oltre ad accrescere e rendere fruibile il valore del patrimonio grazie a interventi progettuali supportati da competenze specifiche di design, ad esempio design dell'allestimento, o design della comunicazione, si può "attivare" il cultural heritage come risorsa (Lupo 2007; Lupo 2011, Lupo 2013a) ovvero usarlo come materia prima di progetto da ricontestualizzare, attualizzare e incorporare in significati, prodotti, processi più vicini alle persone: parafrasando Agamben "profanare per restituire al libero uso degli uomini"(Agamben 2005) in modo culturalmente autentico, consapevole e sostenibile. 


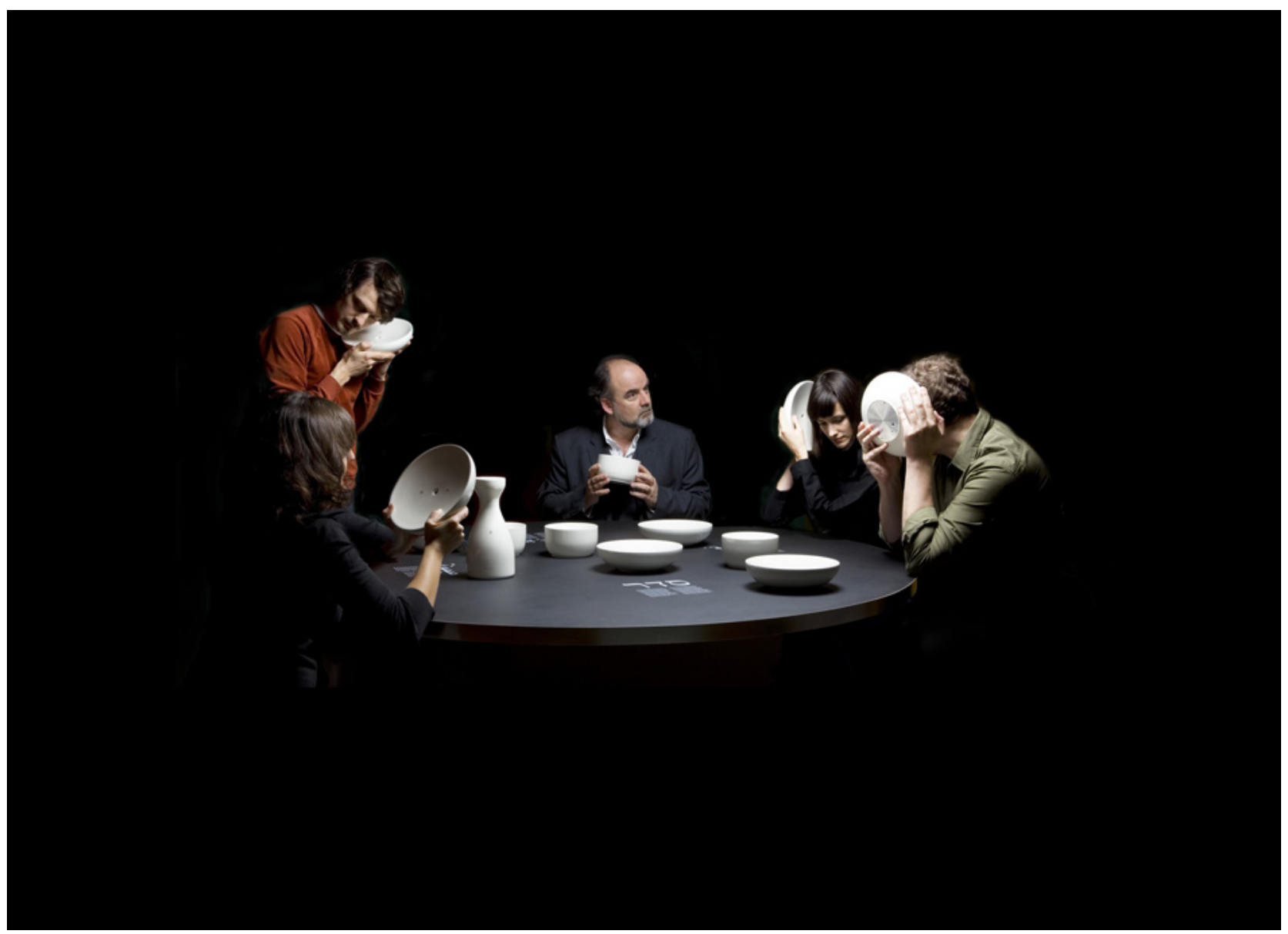

Fig. 1. Installazione Whispering table, Museo Ebraico di Berlino, The Green Eyl, 2009 http://thegreeneyl.com/whispering-table

Il settore dell'intangibile cultural heritage (Unesco, 2003) in particolare si presta a entrambe le interpretazioni progettuali (Lupo 2007, 2011, 2013a, 2013b). Nel primo caso l'esperienza aumentata offerta da apparati allestitivi e tecnologici di tipo interattivo può supportare una migliore comprensione ed appropriazione di beni immateriali quali possono essere tradizioni, rituali, memorie, saperi: esempi autorevoli sono la mostra temporanea "A Oriente. Città uomini e Dei sulle via della seta" (Terme di Diocleziano, Roma, 2011) di Studio Azzurro che attraverso o l'installazione permanente "Whispering table" (Museo Ebraico di Berlino, 2009) di The Green Eyl che permettono ai visitatori, attraverso diversi tipi di interfacce (da TUl- tangible user interfacesa superfici calpestabili), di performare gestualità diverse e non scontate per attivare contenuti culturali (Fig. 1). Nel secondo caso il patrimonio di conoscenze, saperi, tradizioni di una comunità o documentato in una collezione viene riconosciuto come un repertorio di risorse con un reale valore d'uso che può supportare la creatività e una nuova produzione culturale (Cominelli, Greffe, 2012; Sedita 2012) come nel caso del progetto "Inspired by China" (2006) del Peabody Essex Museum (Fig. 2), o "Autentico Contemporaneo Milanese" (2011) del Politecnico di Milano, in cui il cultural heritage diventa un reale 'open-ended knowldge system', ovvero un sistema di conoscenza aperto a molteplici nuovi usi e applicazioni (Lupo et al. 2011). 


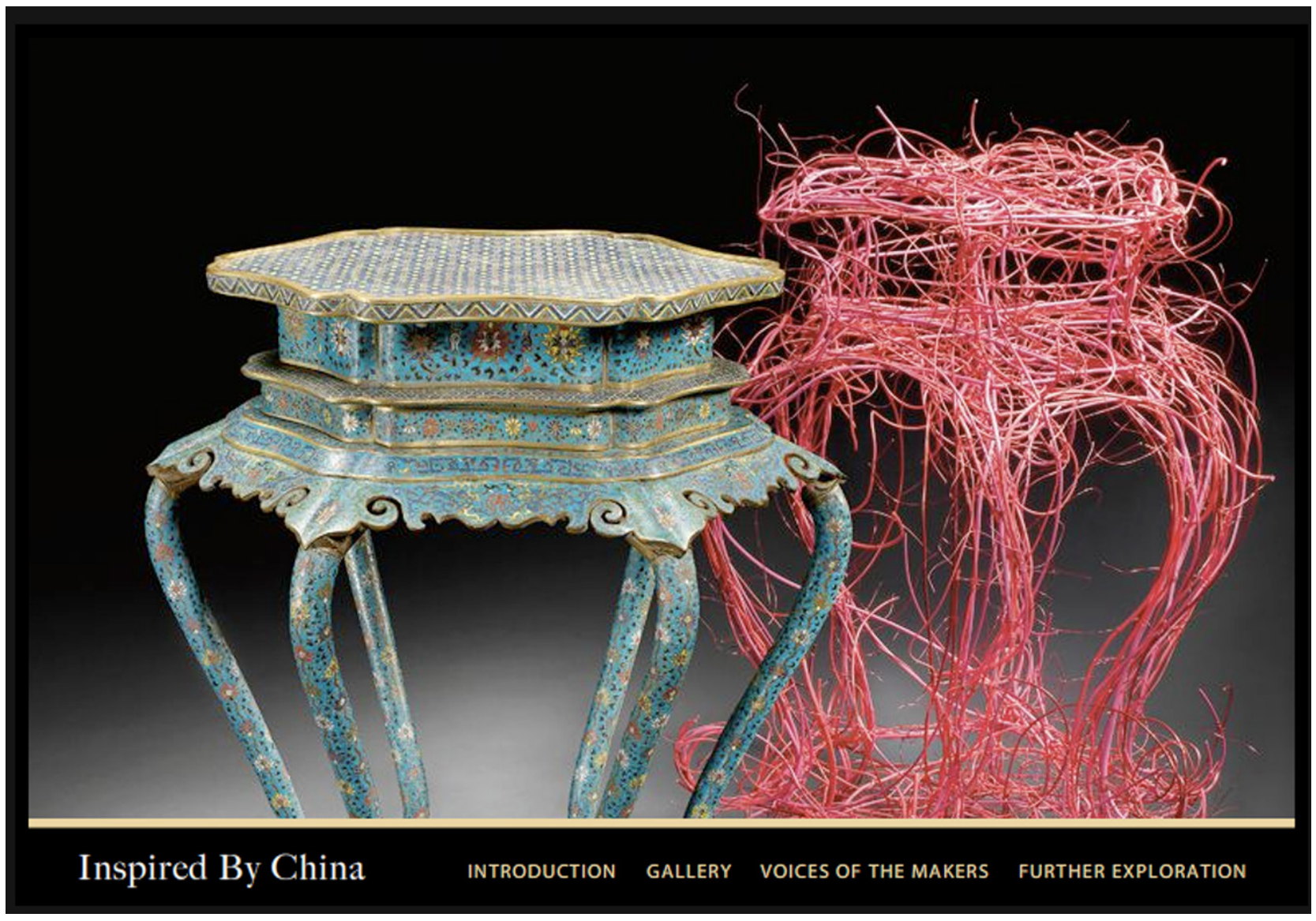

Fig. 2. Mostra Inspired by China, Peabody Essex Museum, Ottobre 2006-Marzo 2007 https://www.pem.org/exhibitions/inspired-by-china-contemporary-furnituremakers-explore-chinese-traditions

\section{Da sviluppo cultural driven a innovazione cultural driven guidata dal design.}

La frontiera sulla quale vogliamo tuttavia spingere questo saggio è quella di portare il design per la valorizzazione dei beni culturali a produrre non ‘solo' sviluppo ma vera e propria innovazione cultural driven.

Il tema della innovazione cultural driven può essere preliminarmente confrontato e associato a tutte quelle esperienze di innovazione sociale dalla forte impronta culturale, ovvero "cultural heritage based/driven social innovation" (Lupo, 2014) che hanno di per se una dimensione sociale legata al valore sociale del cultural heritage e in cui la dimensione culturale è invece rappresentata dall'asset usato (ovvero risorse eminentemente culturali) e dall'obiettivo dell'azione (produzione di cultura o nuovo valore e significato culturale). In queste esperienze l'obiettivo diretto sono l'attivazione e valorizzazione del patrimonio culturale (ai fini di una migliore comprensione o appropriazione, la costruzione di identità e coesione sociale, etc.) e lo sviluppo è un eventuale risultato indiretto di ridistribuzione di valore culturale nel territorio e nelle comunità.

Tuttavia, la conferenza Horizon 2020 "Innovation and Cultural Heritage" tenutasi nel marzo 2018 a Bruxelles, evidenziando come il patrimonio possa svolgere un ruolo di ispirazione creativa per la società, ha declinato 
il concetto di innovazione legato al cultural heritage in termini non solo sociali, ma anche tecnologici, politici, imprenditoriali, economici e metodologici. II report della conferenza sottolinea come anche l'innovazione tecnologica derivata dall'associazione tra patrimonio culturale e nuove tecnologie digitali risulti altrettanto semplicistica di quella sociale, per cui per esempio suggerisce un approccio consapevole alla digitization parlando di culture-driven digitalization vs. technology-driven digitalization. Ma ciò che è maggiormente significativo è la compresenza di diversi piani e livelli di innovazione (politica, imprenditoriale, economica, metodologica, etc.) condensati, secondo il Policy Review della conferenza, in un approccio "olistico" al Cultural Heritage (Sonkoly, Vahtikari, 2018). Si tratta cioè di legare fortemente cultura e impatti in una relazione di causa/ effetto che va oltre il leggere le pratiche creative come pratiche 'anche' culturali (in un ideale allargamento, peraltro già in atto, di cultural heritage inclusivo di forme di produzione culturale contemporanea o di "contemporary heritage"), per, invece, supportare pratiche creative basate direttamente sulla cultura, ovvero sul ri-uso creativo del patrimonio. Inoltre, per avere un impatto reale, occorre re-immaginare tutta la filiera del valore del cultural heritage, progettando non solo offerte culturali per gli utenti finali, ma abilitando anche opportunità per i diversi attori e stakeholders del sistema, quali istituzioni, policy makers, professionisti e imprese culturali e creative.

Perché e come questa innovazione cultural driven può essere efficacemente guidata dal design? Sicuramente perché il design viene riconosciuto non solo come driver di innovazione e sviluppo, ma proprio come espressione di cultura e capacità di proteggere e valorizzare il patrimonio e la diversità culturale: la Montreal Design Declaration ${ }^{2}$ del 2017 sancisce una importante legittimazione del potenziale del design per raggiungere obiettivi economici, sociali, ambientali e culturali a scala globale, attraverso una "call to action" rivolta a professionisti, educatori e istituzioni nel modo del design che non solo indirizza, ma in qualche modo 'impegna' il design a essere vigile, responsabile e soprattutto fattivo come attore culturale in grado di influenzare le politiche pubbliche e l'allocazione delle risorse. Si tratta di una vera e propria design advocacy che ben risponde alle richieste di innovazione olistica del Cultural Heritage espresse dalla comunità europea, in quanto integra finalità di tipo sociale e culturale sia verso la transizione a soluzioni più sostenibili, sia con l'aggiungere valore alle tecnologie.

Sul piano operativo, è possibile delineare dimensioni rilevanti e promettenti di innovazione cultural driven che il design può guidare, in linea con gli ambiti e le metodologie progettuali più attuali (di tipo processuale e metamateriale, partecipativo e co-creativo), e che facciano leva sugli aspetti e valori più "open-ended" del Cultural Heritage (qualità immateriali e loro ri-uso creativo, anche tramite le tecnologie).

\section{Dimensioni d'innovazione Cultural Driven}

II ri-uso creativo del patrimonio culturale è la prima di queste dimensioni di innovazione cultural driven che può essere guidata dal design, trasformando il Cultural Heritage in risorsa per la produzione di nuovi prodotti e servizi culturali.

Alcune esperienze in corso, promosse e finanziate dalla comunità europea a partire da Europeana (la piattaforma digitale commissionata dalla comunità europea con lo scopo di consentire accesso e riuso del patrimonio

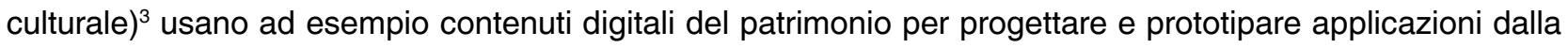
finalità educativa, per il turismo o l'intrattenimento.

\footnotetext{
2 http://www.designdeclaration.org/declaration/

3 https://www.europeana.eu/portal/it
} 
Alcune applicazioni sono pensate per l'insegnamento della storia o per fare ricerca in campo musicale (rispettivamente Historiana ${ }^{4}$ e $\mathrm{MuSO}^{5}$ ) ma anche per seriouys gaming a scopi di intrattenimento educativo (Natural history adventure game ${ }^{6} \mathrm{o}$ Memory Matching game ${ }^{7}$ ).

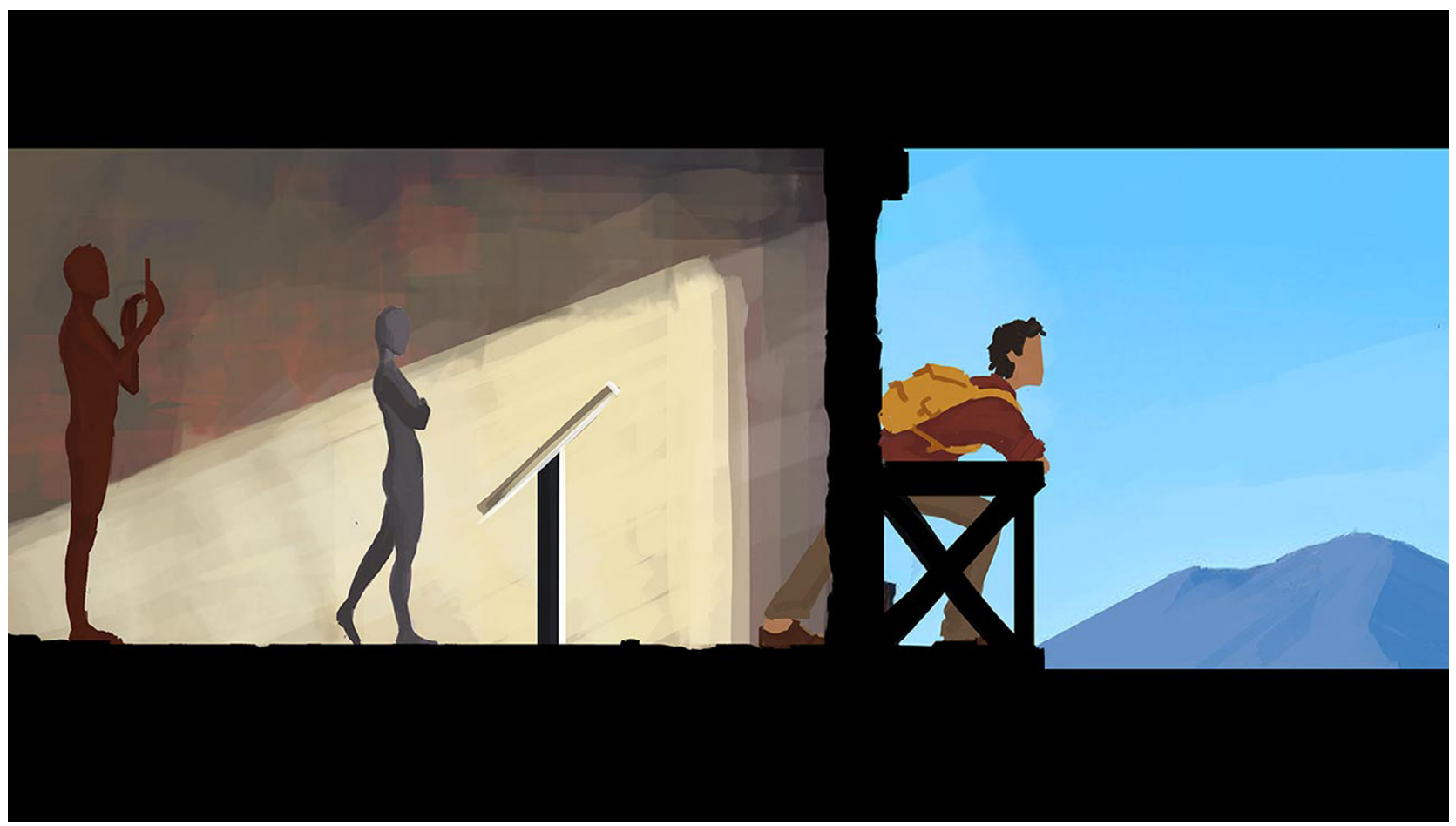

Fig. 3. Videogioco Father and Son, Museo Archeologico di Napoli, Tuo Museo, 2017 http://www.fatherandsongame.com

Un esempio interessante è il videogame Father and Son ${ }^{8}$ sviluppato per il Museo Archeologico di Napoli (MANN), nato con l'idea di allargare il pubblico del museo avvicinando i giovani e basato su un modello innovativo di storytelling, definito storydoing in quanto il fruitore non solo è attivamente coinvolto ma, come protagonista al centro della storia, influenza, con le sue scelte, l'esito finale. Il gioco è ambientato nelle sale del museo e nella città di Napoli e la narrazione si sviluppa in 2D a scorrimento orizzontale con un alto impatto emozionale: avendo ricevuto una lettera dal padre archeologo mai conosciuto, un figlio parte alla scoperta dei suoi appunti ritrovati al MANN, attraversando così diverse epoche storiche, e intrecciando la sua esperienza personale con la Storia collettiva (fig. 3).

In generale è abbastanza evidente che il ri-uso si riferisce principalmente agli aspetti immateriali più che a quelli materiali del patrimonio (non solo in quanto digitalizzati ma perché legati a forme intangibili di $\mathrm{CH}$, quali storia, letteratura, musica, scienza, con i rispettivi significati e valori in termini di conoscenze e applicazioni), perchè possono più facilmente diventare risorse all'interno di pratiche creative basate direttamente sulla cultura,

4 https://pro.europeana.eu/data/historiana

5 https://pro.europeana.eu/data/muso

6 https://pro.europeana.eu/page/natural-history-education-pilots

7 https://pro.europeana.eu/data/memory-match

8 https://www.museoarcheologiconapoli.it/it/father-and-son-the-game/ 
in cui i contenuti culturali non hanno solamente fini educativi, ma sono proprio fonte di ispirazione di nuove applicazioni. Ed appare anche primario il ruolo che assume la tecnologia digitale nell'abilitare questi ri-usi creativi, permettendo azioni di post-produzione, mash-up etc. II ruolo del design in questo è legato sicuramente alla capacità di dare un significato culturale alle soluzioni tecnologiche disponibili, collegandole, grazie ad attività di co-design, a bisogni ed utenti reali, ma soprattutto creando, tramite visioning ed envisioning, connessioni originali con contesti, dinamiche d'uso, ambiti, a volte inaspettati ma di grande opportunità e potenzialità. Ad esempio avvicinando, tramite le tecnologie digitali e mobile, il patrimonio culturale alla quotidianità delle persone, in modo informale e al di fuori dai luoghi deputati della cultura: per poter impersonare opere d'arte (Van Go Yourself ${ }^{9}$ ); creare delle proprie gallerie digitali personalizzare e condividerle sui propri dispositivi (RijskStudio del Rijsk Museum ${ }^{10}$ ), etc.

Perchè si possa raggiungere un'innovazione olistica occorre tuttavia che tali pratiche abbiano la capacità di avere un impatto su tutta la filiera e di coinvolgere in modo sistematico non solo utenti finali, ma tutti i diversi attori e stakeholders del sistema, quali istituzioni, policy makers, professionisti e imprese culturali e creative, oltre il territorio e le comunità interessate.

Quindi, ciò che è veramente fondamentale, come seconda dimensione di innovazione cultural driven che il design può indirizzare, è che tali pratiche di ri-uso creativo non generino solo nuovi prodotti fruibili da utenti finali ma anche meta-output quali elementi e strumenti che gli stessi stakeholders possano utilizzare per produrre a loro volta nuovi ri-usi.

Un esempio è quello di piattaforme on line di archivio di contenuti culturali (curati dalle stesse istituzioni detentrici del patrimonio, quali i musei o gli archivi) che possono essere ricercati tramite varie chiavi per essere riutilizzati a diversi attori per diversi scopi: da artisti per creare altre opere d'arte, da imprese culturali e creative per produrre prodotti culturali tramite diversi media (film, videogames, etc.) e persino prodotti commercializzabili. In questo sicuramente all'avanguardia è il museo olandese Rijsk Museum, che con la piattaforma on line RijskStudio consente non solo, aprendo un proprio profilo, di creare gallerie personali di immagini e acquistare stampe ad alta risoluzione delle stesse, ma soprattutto di scaricare immagini ad altissima risoluzione per poterle applicare ad altri contesti. Le immagini (dipinti, disegni, oggetti) sono ricercabili attraverso diversi criteri, tra cui (oltre i classici autore, tipologia, etc.) alcuni (ad es. la palette cromatica di riferimento, o il tema, o elementi raffigurati anche in dettagli), sono emblematici della scelta di supportare ricerche meno tradizionali e quindi connessioni tematiche sulla base dell'intenzione d'uso dell'utilizzatore. Le immagini sono rilasciata tutte con licenza d'uso aperta ("pubblico dominio" o "no copyright"11) per cui possono essere copiate, modificate, distribuite anche per usi commerciali senza chiedere alcun permesso: la stessa piattaforma suggerisce con degli esempi i possibili ri-usi e chiede che vengano condivisi on line per ispirare altri utenti (fig. 4). Questo approccio di patrimonio come fonte di ispirazione inizia ad esser condiviso da tantissimi musei e istituzioni culturali anche se con diversi livelli di licenze d'uso (aperte o meno): ad esempio il Moma rende disponibili alcune immagini di alcune mostre $^{12}$ ma occorre chiedere un permesso formale per la riproduzione (al Moma o al detentore del copyright, ovvero spesso l'autore, dell'opera).

9 https://vangoyourself.com

10 https://www.rijksmuseum.nl/en/rijksstudio

11 https://creativecommons.org/publicdomain/zero/1.0/deed.en

12 https://www.moma.org/calendar/exh ibitions/history?locale=en 


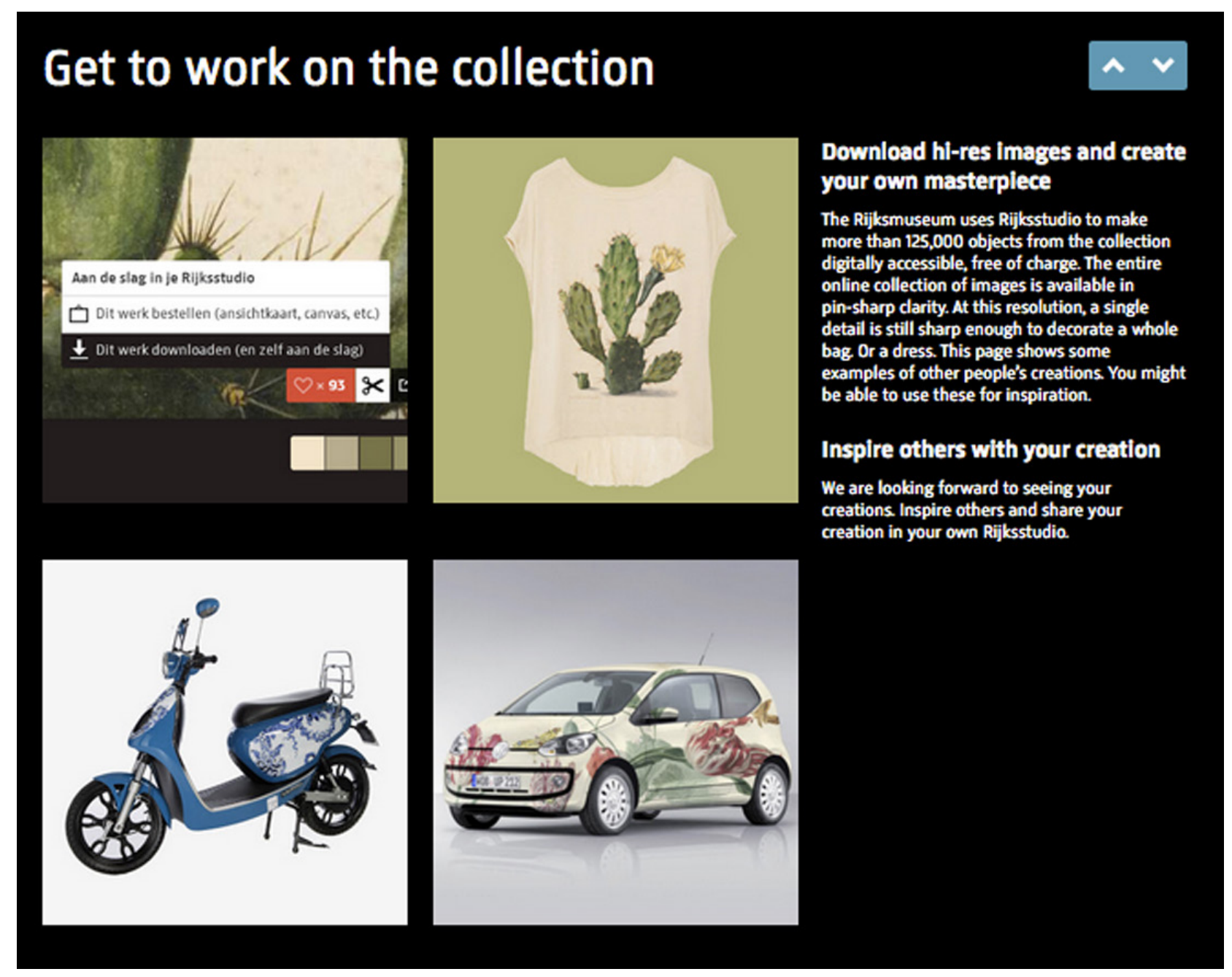

Fig. 4. Sito web Rijsk Studio, Rijsk Museum https://www.rijksmuseum.nl/en/rijksstudio

Il ruolo del design in questo è proprio quello di creare un modello sostenibile di co-creazione di contenuti culturali, che superi il semplice concetto di partecipazione al patrimonio. I musei sono ormai largamente impegnati nell'offrire, grazie, ma non solo, all'uso di tecnologie digitali e mobile, esperienze personalizzate, interattive e contributive (Allen, Lupo, 2012), per cui II ruolo attivo del fruitore dei contenuti culturali si esprime non solo nelle dinamiche di visita ma anche nel poter aggiungere e condividere contenuti attraverso strategie di co-curatela: approcci quali seriuos interpretation consentono di generare user generated contents (UGC) rilevanti (culturalmente consistenti) e autorevoli, per garantire la qualità dell'esperienza (Lupo 2019- in press). Il concetto di co-creazione supera tuttavia anche il modello di co-curation dei participatory museums 13 perché letteralmente apre la disseminazione dei contenuti culturali in applicazioni totalmente al di fuori dei contesti istituzionali, persino commerciali, in un cambio di prospettiva a volte ancora difficile da accettare per le istituzioni culturali stesse, ma che sta avvenendo a prescindere dalla loro volontà. È lo stesso continuum 
(citato a inizio saggio) che esiste tra forme di Heritage autorevole e forme di heritage contemporaneo e diffuso a offrire il contesto favorevole a questo cambiamento.

Cruciale è quindi il ruolo del design nell'abilitare e potenziare sistemi e tecnologie di accesso e riutilizzo sostenibile del patrimonio, ma soprattutto nel comunicare e rendere quindi visibile, comprensibile e condivisibile il valore di questo cambio di prospettiva ai detentori del patrimonio e i suoi potenziali utilizzatori, evitando in questo campo atteggiamenti stereotipati e semplificatori quali toolification e solutionism (Manzini, 2006), ovvero un approccio alle tecnologie di tipo esclusivamente strumentale, per promuoverne invece uno realmente politico e culturale.

Si inserisce quindi qui il contributo che il design può offrire, oltre che nella progettazione della usabilità di piattaforme di ricerca di contenuti culturali (di cui abbiamo offerto sopra alcuni esempi attraverso per esempio interfacce che consentano di ricercare e confrontare per colori o soggetti, etc.), quello relativo alla progettazione di applicazioni (API- application programming interfaces, ad esempio quella di Europeana ${ }^{14}$ ) per il riutilizzo dei dati culturali da parte di programmatori, fino alla concezione di nuove metrics, policies e standards (anche in linea con quanto espresso nella Montreal Design Declaration sopracitata) per il riutilizzo del Cultural Heritage, quali ad esempio quelle per la gestione dei diritti di proprietà intellettuale (IPR).

Come abbiamo visto infatti, il fatto che tanti contenuti culturali siano disponibili in forma digitale in rete non implica che siano anche direttamente ri-utilizzabili da chiunque: la piattaforma di Europeana ad esempio permette di cercare direttamente immagini attraverso le licenze d'uso attribuite dal detentore dei diritti ${ }^{15}$ (dal riutilizzo gratuito a quello limitato, fino a nessun ri-utilizzo consentito). Anche il sito di Creative Commons ${ }^{16}$ specifica già in dettaglio il tipo di ri-uso possibile dei contenuti culturali al suo interno.

Anche in questo caso il valore del contributo del design non deve però essere semplicemente strumentale nel progettare più efficienti sistemi e tecnologie abilitanti (ad esempio per l'affinamento della ricerca tramite IPR o la comprensione dei criteri IPR) quanto di sollecitare, coinvolgendo tutti gli stakeholder, attraverso processi altrettanto partecipati, la riflessione sui modelli di proprietà intellettuale, proponendo possibili visioni alternative e complementari. Ad esempio, il progetto europeo RICHES ${ }^{17}$ ha promosso un interessante dibattito su questo tema suggerendo il passaggio da un modello di copyright (che vede il $\mathrm{CH}$ come un asset) a uno di human right (che vede il $\mathrm{CH}$ anche come risorsa) ${ }^{18}$. Anche la possibilità di offrire dei corsi on line aperti a tutti (come il MOOC-massive on line open course- di Europeana Space "Creative with Cultural Heritage"19) può essere uno strumento per promuovere conoscenza sulle possibilità di riuso del $\mathrm{CH}$.

Una nuova consapevolezza deve essere quindi socialmente e culturalmente, ma anche politicamente e legalmente condivisa, sul concetto di autenticità e tracciabilità dei contenuti culturali al fine di consentirne un vero ri-uso, e il design può, attraverso le sue competenze di progettazione di processi di trasformazione

\footnotetext{
14 https://pro.europeana.eu/resources/apis

15 https://www.europeana.eu/portal/it/search?f\%5BTYPE\%5D\%5B\%5D=IMAGE\&per page=72\&q=\&view=grid\&f\%5BREUSABILITY\%5D\%5B\%5D=open

16 https://search.creativecommons.org/

17 https://www.riches-project.eu/

18 Documenti specifici del progetto sul tema sono disponibili alla pagina: https://resources.riches-project.eu/category/research-field/ digital-copyright/
}

19 https://www.edx.org/course/europeana-space-creative-with-digital-heritage 
collettiva, la sua vocazione verso le componenti immateriali del progetto e la sua advocacy di attore culturale, costruire il solido ponte che lega tecnologie e tutte le forme attuali di Cultural Heritage, in un modello di innovazione cultural driven, promuovendo la valorizzazione tramite un ri-uso sostenibile del patrimonio che coinvolga tutti gli stakeholders attraverso non soltanto nuovi prodotti culturali ma strategie e strumenti di cocreazione per la filiera.

\section{Bibliografia}

Affleck J., Kvan, T. (2008). Memory Capsules. Discursive Interpretation of Cultural Heritage through New Media. In: J. Afflek, Y. E. Kalay, T. Kvan (a cura di) New Heritage. New Media and Cultural Heritage. London and New York: Routlege.

Agamben, G. (2006). Che cos'e un dispositivo? Roma: Nottetempo

Allen J., Lupo, E. (a cura di). (2012). Representing Museum Technologies. Copenhagen: Copenhagen Interaction Design Institute

Battesti, J. (a cura di). (2012). Que reste-t-il du present? Collecter le contemporain dans le musees de societe. Bordeaux: Le Festin

Cabrera-Viancha, P. (2005). Design, craft, artisan communities: sustainable development of cultural diversity. In: E. Corte-Real, A. M. C. Duarte, F. Carvallo Rodrigues (a cura di) Pride and predesign. The cultural Heritage and the science of design. Lisbon: Unicom.

Celaschi, F., Trocchianesi, R. (a cura di). (2004). Design e beni culturali. La cultura del progetto nella valorizzazione del bene culturale. Milano: Poli.design

Chih-Yuan, C. (2005). The rebirth of the landscape cultural heritage. In: E. Corte-Real, A. M. C. Duarte, F. Carvallo Rodrigues (a cura di) Pride and predesign. The cultural Heritage and the science of design. Lisbon: Unicom.

Cominelli, F. Greffe, X. (2012). Intangible cultural heritage. Safeguarding for creativity. City, Culture and Society 2: $245-250$

Corte-Real, E., Duarte, A. M. C., Carvallo Rodrigues F. (a cura di) Pride and predesign. The cultural Heritage and the science of design. Lisbon: Unicom

Dernie, D. (2006). Exhibition Design. London: Laurence King Publishing

Giaccardi, E. (a cura di).(2012). Heritage and Social Media: Understanding Heritage in a Participatory Culture. London and New York: Routledge

Greffe, X. (2005). Cultura e sviluppo locale. Trento: Nicolodi

Kuo Wai Tchen, J., Ševcenko L. (2011). The 'dialogic museum' revisited: a collaborative reflection. In: B. Adair, B. Filene, L. Koloski (a cura di). Letting go. Sharing Historical Authority in a User-Generated World. Philadelphia: The Pew Center for Arts \& Heritage

Lupo, E. (2007). Intangible Heritage valorisation: a new field for design research and practice. In: S. Poggenpohl (a cura di) Proceeding of the IASDR 07 Conference. Hong Kong: School of design, Honk Kong Polytechnic University

Lupo, E. (2011). Design, beni culturali immateriali e 'attivazione dell'autentico': progettare il valore delle eredità culturali come 'open-ended knowledge system'. In: i+diseno 04, pp. 46-56

Lupo E. (2013a). Percorsi e scenari dell'intangible. Le filiere del progetto e il potenziale di innovazione del patrimonio immateriale come 'open-ended knowledge system'. In: Irace F. (a cura di). L'immateriale, il virtuale, l'interattivo. Milano: Modadori Electa. 
Lupo E. (2013b). Forme dell'Heritage, forme del progetto: saperi tipici e dinamiche di 'attivazione' design driven. In: E. Lupo (a cura di) Autentico Contemporaneo Milano. Design e attivazione dei saperi tipici e maestri artigiani milanesi. Milano: Maggioli.

Lupo, E. (2014). Beyond Social Innovation. Design as Cultures Active-action. In: C. Coletta, S. Colombo, P. Magaudda, A. Mattozzi, L.L. Parolin and L. Rampino (a cura di). A Matter of Design. Proceedings of the V STS Italia Conference. Milano: STS Italia

Lupo, E. et al. (2011). Design research and cultural heritage: activating the value of cultural assets as "openended knowledge system". Design Principles and Practices Journal, 5 (6), pp.431- 450

Lupo, E., Vitale, G. (2017). Design strategies for Cultural Heritage. Innovating tradition in Museums. In: G. Amoruso (a cura di) Putting tradition into Practice: Heritage Places and Design, Proceedings of 5th INTBAU International Annual Event. Cham: Springer

Maffei, S., Parente, M., Villari, B. (a cura di). (2006). SDI Design Review 03: II design per la valorizzazione dei beni culturali. Le premesse fondative della ricerca d.Cult. Milano: Poli.design

Manzini, E. (2006). Design culture and dialogic design. Design Issues, 32 (1), pp. 52-59.

Ozdil, E. (2014). Archives as new spaces for engaging experiences: Technologies and Languages within the Scenario of "Heritage Continuum". In: H. Gottlieb, M. Szelag (a cura di), Engaging Spaces - Interpretation, Design and Digital Strategies. Proceedings of the NODEM 2014 International Conference. Warsaw, Poland: Interactive Swedish Institute ICT

Parente, M., Lupo, E. (a cura di). (2009). II Sistema Design Italia per la valorizzazione dei beni culturali. Milano: Poli.design

Perrone, R., Büchler, D. (2005). Identity and memory. In: E. Corte-Real, A. M. C. Duarte, F. Carvallo Rodrigues (a cura di) Pride and predesign. The cultural Heritage and the science of design. Lisbon: Unicom.

Pombo, F. (2005). Design as maker of cultural survival. In: E. Corte-Real, A. M. C. Duarte, F. Carvallo Rodrigues (a cura di) Pride and predesign. The cultural Heritage and the science of design. Lisbon: Unicom.

Salgado, M., Marttila, S. (2013). Discussion on inclusive, participative and open museums. In: H. Gottlieb (a cura di), Beyond control: the collaborative museums and its challenges. Proceedings of the NODEM 2013 International Conference on Design and Digital Heritage, Stockholm, Sweden: Interactive Swedish Institute ICT.

Seassaro, A. (2003). II design dei beni culturali come estetica della città nella "Milano capitale del design". In Manzocchi, G., Villani, A., Sulla citta, oggi. Arte, beni culturali, istituzioni. Milano: Franco Angeli.

Sedita, S.R. (2012). Leverging the intangible cultural heritage: novelty and innovation trough exaptation. In: City, Culture and Society, 2, 251-259

Simon, N.( 2010). The Participatory Museum. Santa Cruz: Museum 2.0

Smith, R. C., a Iversen,O.S. (2014) .Participatory Heritage Innovation: Designing Dialogic Sites of Engagement. In: Digital Creativity Journal 25 (3), p. 255-268

Sonkoly, G., Vahtikari, T. (2018). Innovation in Cultural Heritage research. Fora $n$ integrated European research policy. Brussels: European Commission Directorate-General for Research and Innovation

Studio Azzurro (2011). Musei di narrazione. Percorsi interattivi e affreschi multimediali. Milano, Silvana Editoriale, $192 \mathrm{p}$.

UNESCO (2003). Convention for the safeguarding of Intangible Cultural Heritage. Available at: https://ich. unesco.org/en/convention Accessed on: January, 2019 
Vasconcellos, P.V. (2005). Rebirth: how ancient art forms are translated and revitalized through their use in modern media. In: E. Corte-Real, A. M. C. Duarte, F. Carvallo Rodrigues (a cura di) Pride and predesign. The cultural Heritage and the science of design. Lisbon: Unicom.

Wood, N. (2005). Unknown knows: uncovering tacit knowledge for the design of interactive media. In: E. Corte-Real, A. M. C. Duarte, F. Carvallo Rodrigues (a cura di) Pride and predesign. The cultural Heritage and the science of design. Lisbon: Unicom. 